\title{
CAPÍTULO 34: PERFIL DOS CONSUMIDORES DE KOMBUCHA
}

\section{CHAPTER 34: PROFILE OF KOMBUCHA CONSUMERS}

\author{
Sonara de França Sousa ${ }^{1}$; Larissa Nunes Torres²; Williames Fabio de Souza Bezerra Filho ${ }^{3}$; Maria Clara Leal \\ Bezerra Barros ${ }^{4}$
}

\begin{abstract}
Resumo
Objetivou-se com o presente trabalho caracterizar o perfil dos consumidores de kombucha por meio de um questionário online. O questionário continha 13 perguntas de múltipla escolha relacionadas aos aspectos socioeconômicos e ao padrão de consumo, tais como: se fazem o uso de bebidas alcoólicas; a frequência e o tipo de bebida mais consumida; se conhecem uma bebida probiótica; se já ouviram falar sobre kombucha; se já consumiram e sabem dos benefícios associados à esta bebida; se conhecem o processo de fabricação da kombucha e se a mesma é comumente encontrada no mercado de sua região. De acordo com os resultados obtidos, os respondentes são em sua maioria mulheres, entre 21 e 30 anos, apresentam pós-graduação e renda mensal entre 1 e 2 salários mínimos. A maioria dos participantes fazem o uso de álcool $(66,4 \%)$ ao menos uma vez por semana, sendo a cerveja a bebida mais consumida $(55,7 \%)$. A maioria dos respondentes afirmaram saber o que é uma bebida probiótica e conhecerem os benefícios associados à kombucha, no entanto, essa bebida não apresenta uma disponibilidade no mercado consumidor $(79,77 \%)$ apesar de se mostrar bastante promissora no segmento de alimentos funcionais.
\end{abstract}

Palavras-Chaves: bebidas probióticas, mercado consumidor, saúde.

\begin{abstract}
The objective of this study was to characterize the profile of kombucha consumers through an online questionnaire. The questionnaire contained 13 multiple-choice questions related to socioeconomic aspects and consumption patterns, such as: whether they use alcoholic beverages; the frequency and type of drink most consumed; if they know a probiotic drink; if you've heard of kombucha; if they have already consumed and know the benefits associated with this drink; the kombucha manufacturing process is known and whether it is commonly found in the market in your region. According to the results obtained, the respondents are mostly women, between 21 and 30 years old, have postgraduate studies and monthly income between 1 and 2 minimum wages. Most participants use alcohol (66.4\%) at least once a week, with beer being the most consumed beverage (55.7\%). Most respondents stated that they know what a probiotic drink is and know the benefits associated with kombucha, however, this drink does not have an availability in the consumer market (79.77\%) despite being very promising in the functional food segment.
\end{abstract}

Keywords: probiotics drinks, consumer market, health.

1Doutora em Engenharia de Processos, Universidade Federal do Agreste de Pernambuco, sonara_franca@yahoo.com.br

2Engenharia de Alimentos, Universidade Federal de Agreste de Pernambuco, larissan.torres159@ gmail.com

3Engenharia de Alimentos, Universidade Federal de Agreste de Pernambuco, williamesfabio_2001@outlook.com

4Engenharia de Alimentos, Universidade Federal do Agreste de Pernambuco, mclaraleal.engalimentos@gmail.com 


\section{Introdução}

De acordo com a Portaria $\mathrm{n}^{\circ} 103$, de 20 de setembro de 2018, a kombucha é definida como sendo uma bebida gaseificada, não pasteurizada, obtida através da respiração aeróbica e fermentação anaeróbica de um mosto composto de infusão de origem vegetal e açúcares por um consórcio de bactérias e leveduras simbióticas microbiologicamente ativas, resultando em uma bebida ácida e doce (BRASIL, 2018).

Segundo Santos (2016), essa bebida era produzida há cerca de 221 anos a.C e conhecida popularmente como o "chá da imortalidade". A origem da kombucha é incerta e acredita-se que tenha surgido no nordeste da China, mais precisamente em Manchúria, já a origem do seu nome veio devido a um médico que se chamava Kombu e que levou a bebida da Coréia para o Japão para curar problemas digestivos de um Imperador e daí o nome "Kombu Chá" ou "Chá de Kombu".

Desde o seu descobrimento, existem relatos demonstrando que essa bebida pode auxiliar na redução do risco de doenças crônicas e também possui propriedades curativas, porém só na última década é que se observou uma explosão em sua popularidade, principalmente em países como os Estados Unidos, que atualmente possui um mercado bem estabelecido para esse produto (SANTOS, 2016). Já no cenário nacional, a kombucha é produzida de forma artesanal, por pessoas que se interessaram em desenvolver a bebida em casa, e só há cerca de 3 anos, indústrias de pequeno porte começaram a produzir a bebida para venda em mercados regionais. A kombucha tem sido bem aceita pelos consumidores, especialmente como alternativa ao refrigerante por ser gaseificada, conter baixo teor de açúcar e poucas calorias (CITRUS, 2018).

Diante disso, objetivou-se com o presente trabalho caracterizar o perfil dos consumidores de kombucha por meio de um questionário online, visando obter informações sobre seus hábitos, motivações, conhecimento e disponibilidade da bebida no mercado local.

\section{Material e Métodos}

Essa pesquisa foi realizada por meio de um questionário online elaborado na plataforma Google ${ }^{\circledR}$ Forms, compartilhado nas redes sociais via link a um grupo de 262 pessoas que se autodeclaram serem maiores de 18 anos.

O questionário continha 13 perguntas de múltipla escolha relacionadas aos aspectos socioeconômicos dos respondentes (gênero, faixa etária, escolaridade e renda mensal) e ao padrão de consumo, tais como: se fazem o uso de bebidas alcoólicas, a frequência do uso de bebidas alcoólicas e o tipo de bebida mais consumida, sendo estas duas últimas perguntas respondidas apenas pelos os que afirmaram fazer o uso de álcool. 
Todos os participantes responderam ainda se eles conheciam o conceito de uma bebida probiótica, se já ouviram falar em kombucha, se já consumiram a kombucha e sabiam dos benefícios associados à esta bebida, se conhecem o processo de fabricação da kombucha e se a mesma é comumente encontrada no mercado de sua região.

Os dados obtidos foram dispostos em uma planilha eletrônica em forma de banco de dados (Excel for Windows), analisados quantitativamente e os resultados dispostos em forma de gráficos, sendo discutidos conforme os tópicos pesquisados.

\section{Resultados e Discussão}

De acordo com os resultados apresentados nas Figuras 1, 2, 3 e 4, a maioria dos indivíduos eram do sexo feminino (82,10\%), com faixa etária entre 21 e 30 anos (40,5\%), nível de escolaridade pós-graduação $(33,6 \%)$ e apresentavam renda mensal entre 1 e 2 salários mínimos $(31,3 \%)$.

$\mathrm{Na}$ literatura disponível não foi encontrado trabalhos relacionados aos perfis dos consumidores de kombucha, sendo os dados comparados à outros tipos de bebidas, como por exemplo, Yassin (2019), analisando o perfil de consumo de bebidas do tipo smoothies, verificou que, dos 500 voluntários que responderam o questionário online, 84,2\% eram do sexo feminino, com idade entre 19 e 35 anos (74,2\%) e renda familiar mensal de 4 a 10 salários mínimos (48\%). Já segundo Martins (2019), analisando o comportamento de 82 indivíduos consumidores de cervejas artesanais no município de Santana do Livramento (RS), observou que $76 \%$ eram do sexo masculino, com idade entre 26 a 35 anos (48\%), apresentavam ensino superior completo (44\%) e renda mensal entre 2.500 a 5.000 reais (39\%). 
Figura 1. Gênero dos participantes que responderam o questionário.

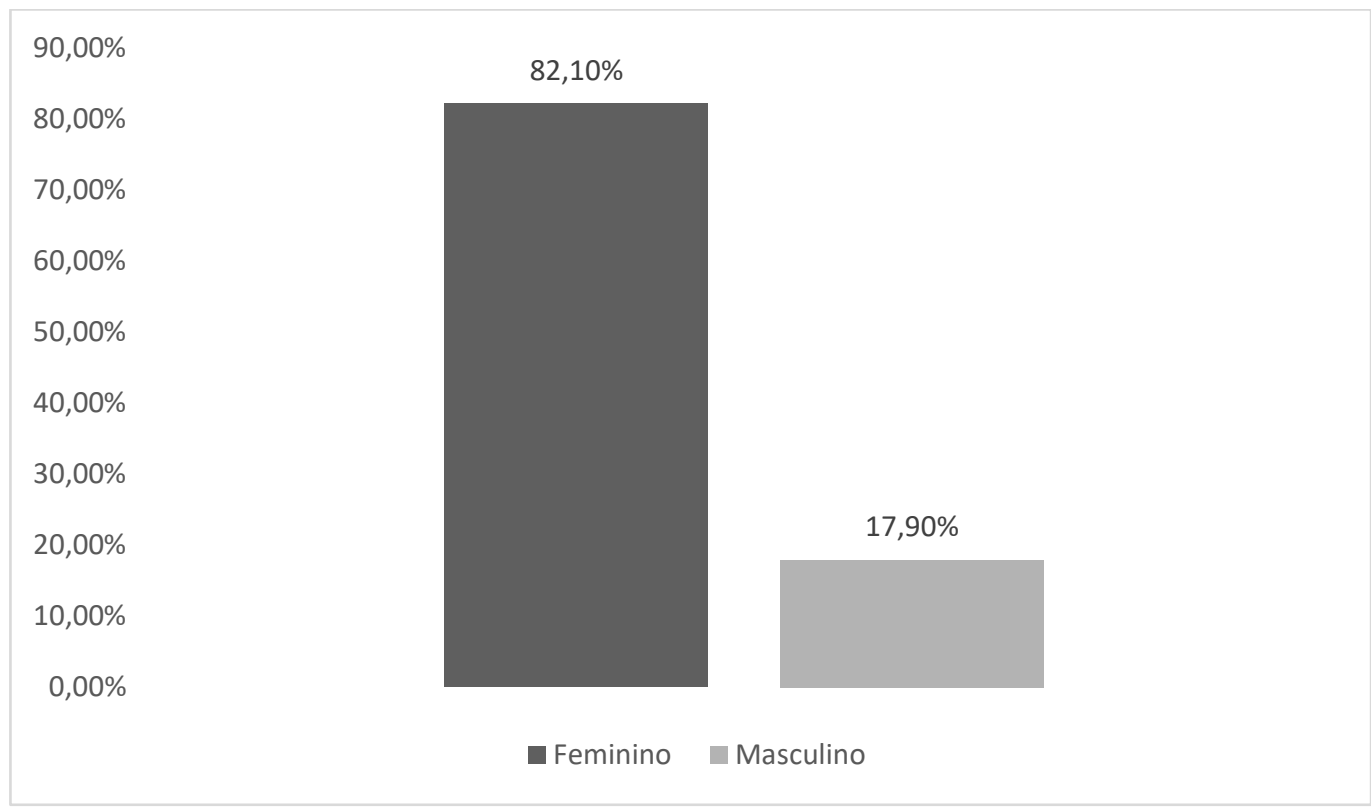

Fonte: Própria (2020)

Figura 2. Faixa etária dos participantes que responderam o questionário.

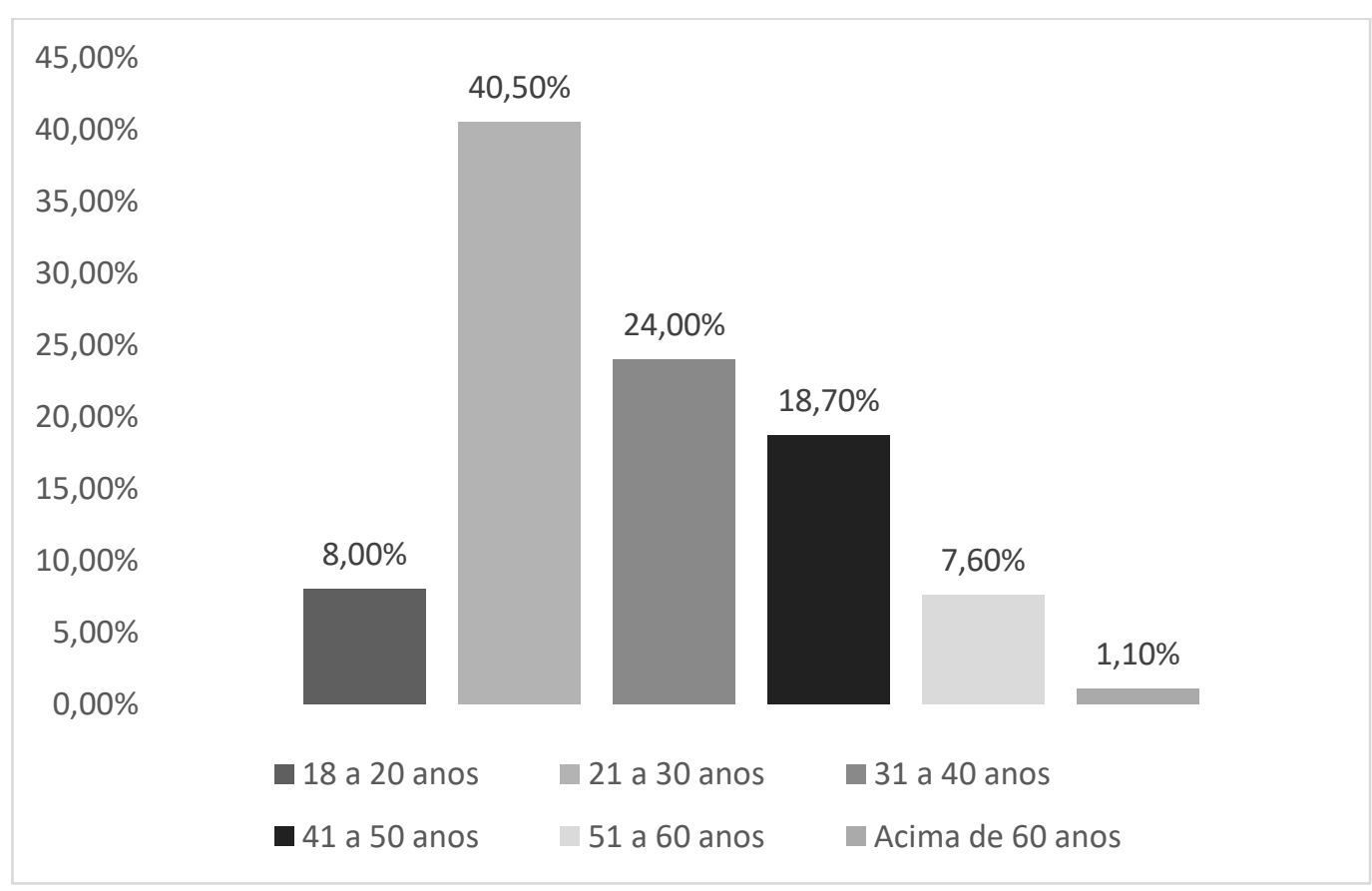

Fonte: Própria (2020) 
Figura 3. Nível de escolaridade dos participantes que responderam o questionário.

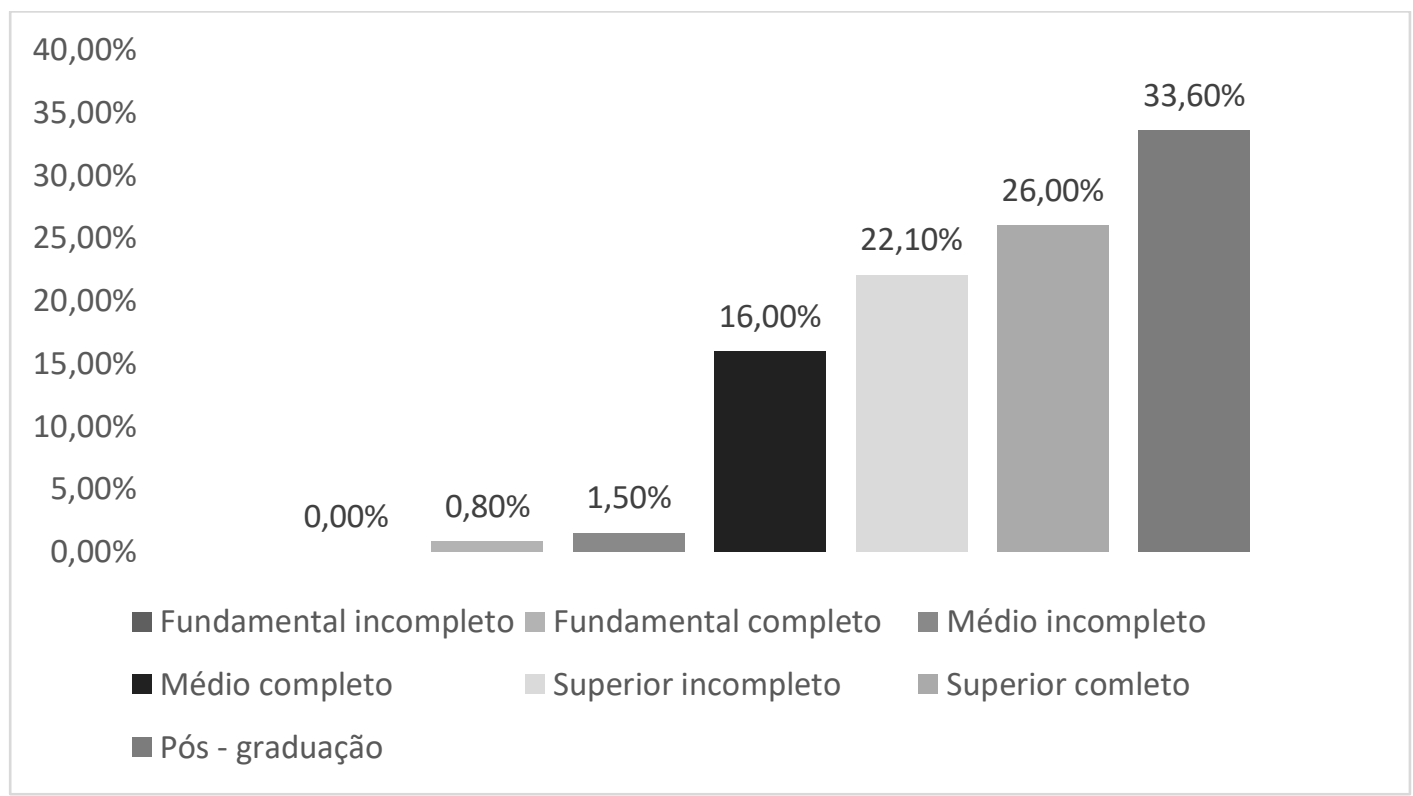

Fonte: Própria (2020)

Figura 4. Renda mensal dos participantes que responderam o questionário.

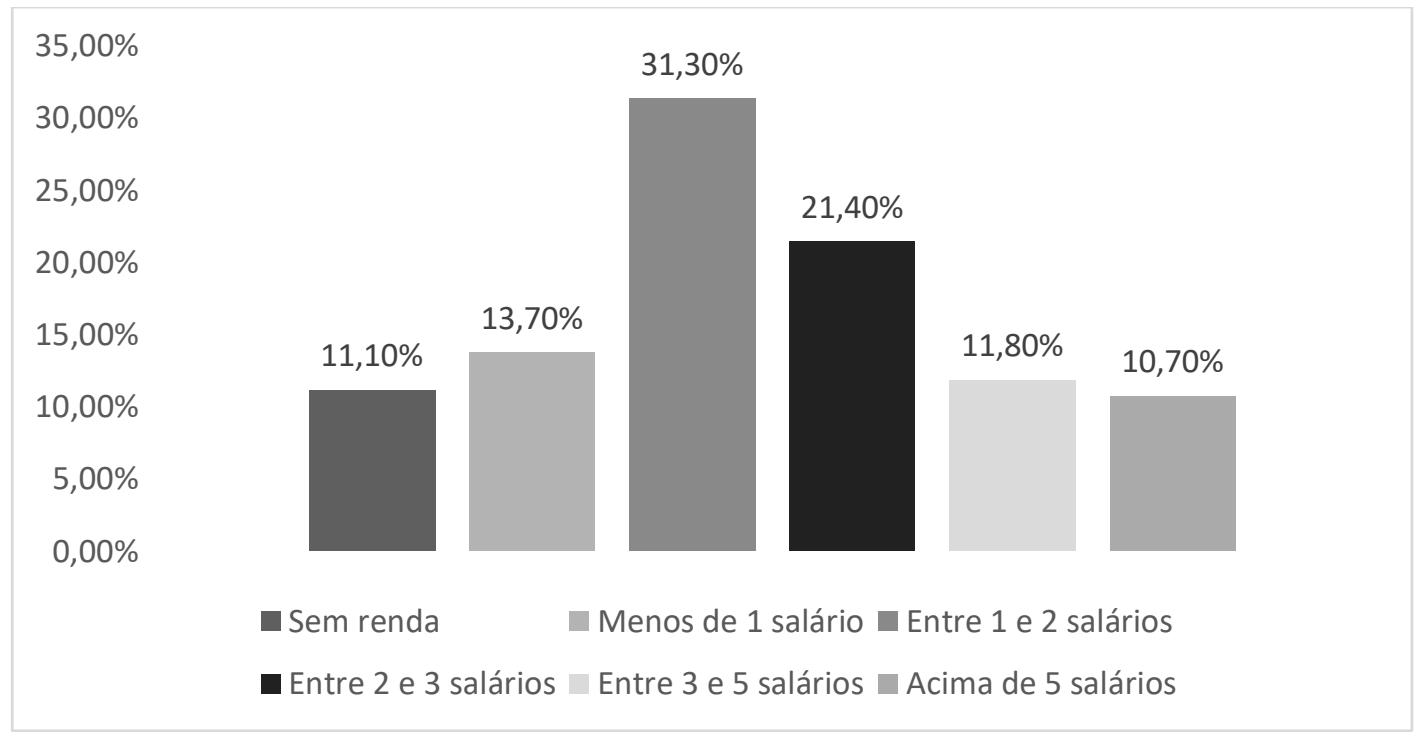

Fonte: Própria (2020)

Na Figura 5 foi perguntado aos participantes se eles fazem o uso de bebidas alcoólicas, $66,4 \%$ responderam que sim. A kombucha pode ser classificada como sendo uma bebida não alcoólica (até 0,5\% de teor alcoólico) e alcoólica (mais de 0,5\% de teor alcoólico) segundo a Instrução Normativa n 41 de 2019 (BRASIL, 2020). De acordo com Chen e Liu (2000), a concentração de etanol no kombucha aumenta com o tempo de fermentação, atingindo valor máximo aproximado de 5,5 g/L no vigésimo dia de fermentação, seguida de uma redução lenta. Sua produção ocorre através da fermentação da glicose, e posteriormente sofre uma segunda oxidação para produzir ácido acético. 
Figura 5. Participantes que fazem ou não o uso de bebidas alcoólicas.

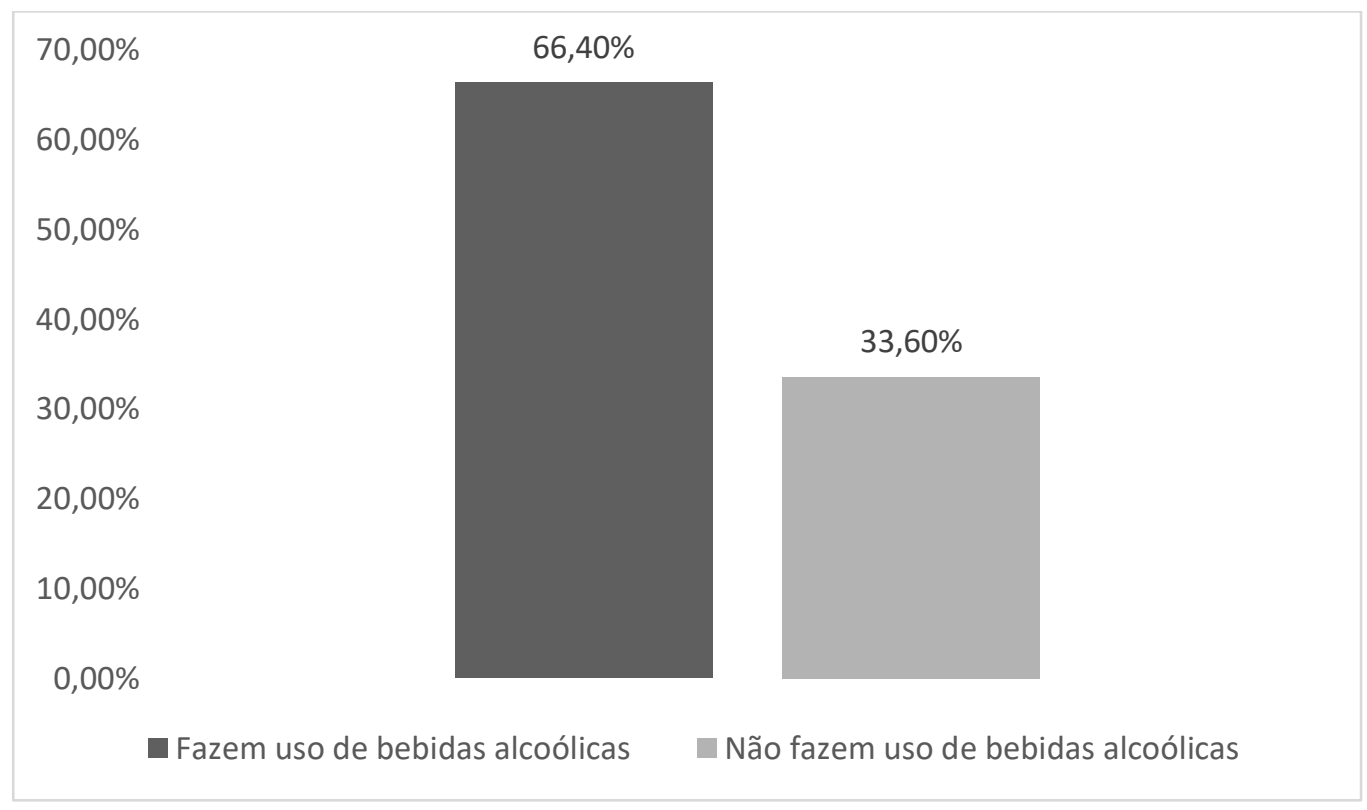

Fonte: Própria (2020)

As Figuras 6 e 7 referem-se à frequência e ao tipo de bebidas alcoólicas mais consumidas, respectivamente, estas por sua vez, foram respondidas apenas pelos usuários que afirmaram ser consumidores de bebidas alcoólicas, conforme o item anterior (Figura 5).

Figura 6. Frequência do consumo de bebidas alcoólicas.

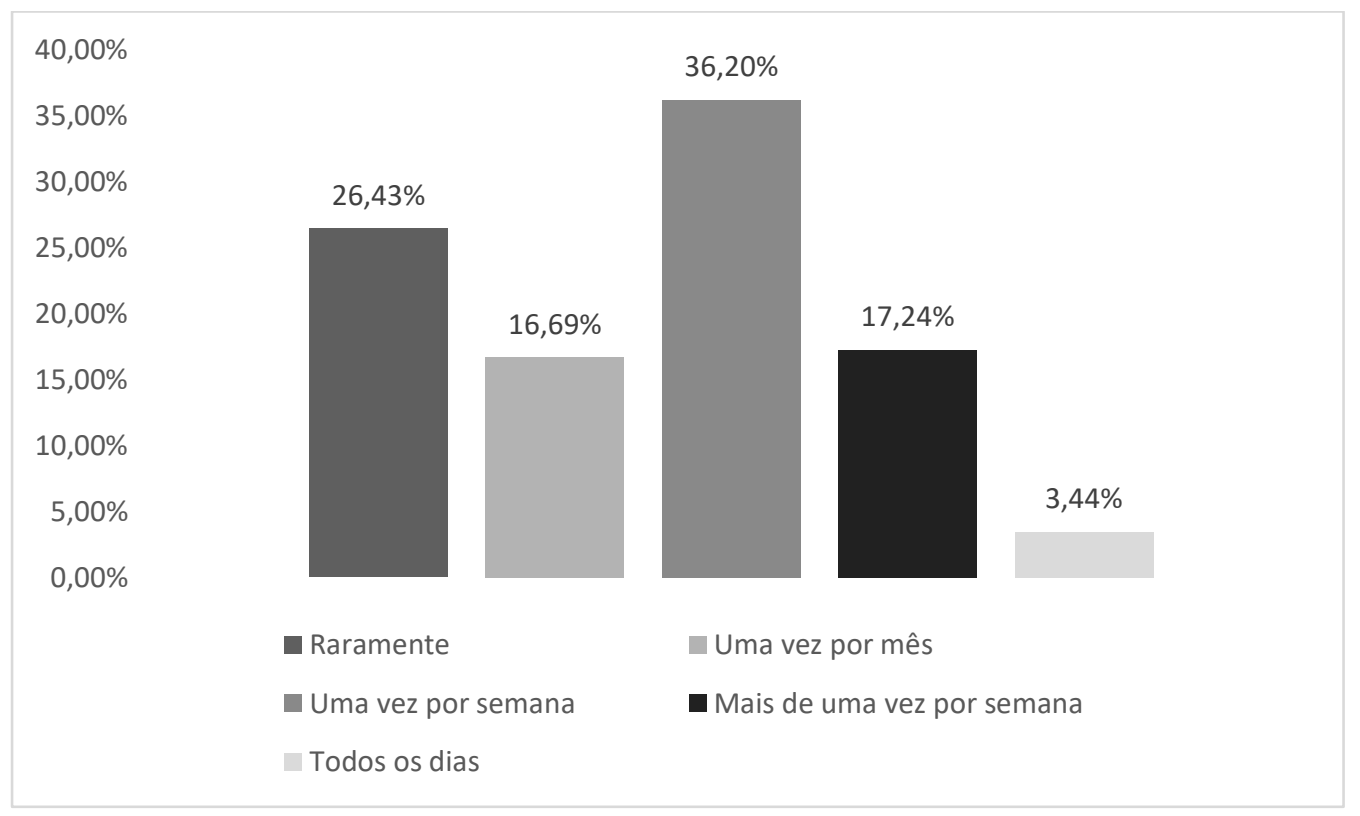

Fonte: Própria (2020)

Figura 7. Tipos de bebidas mais consumidas. 


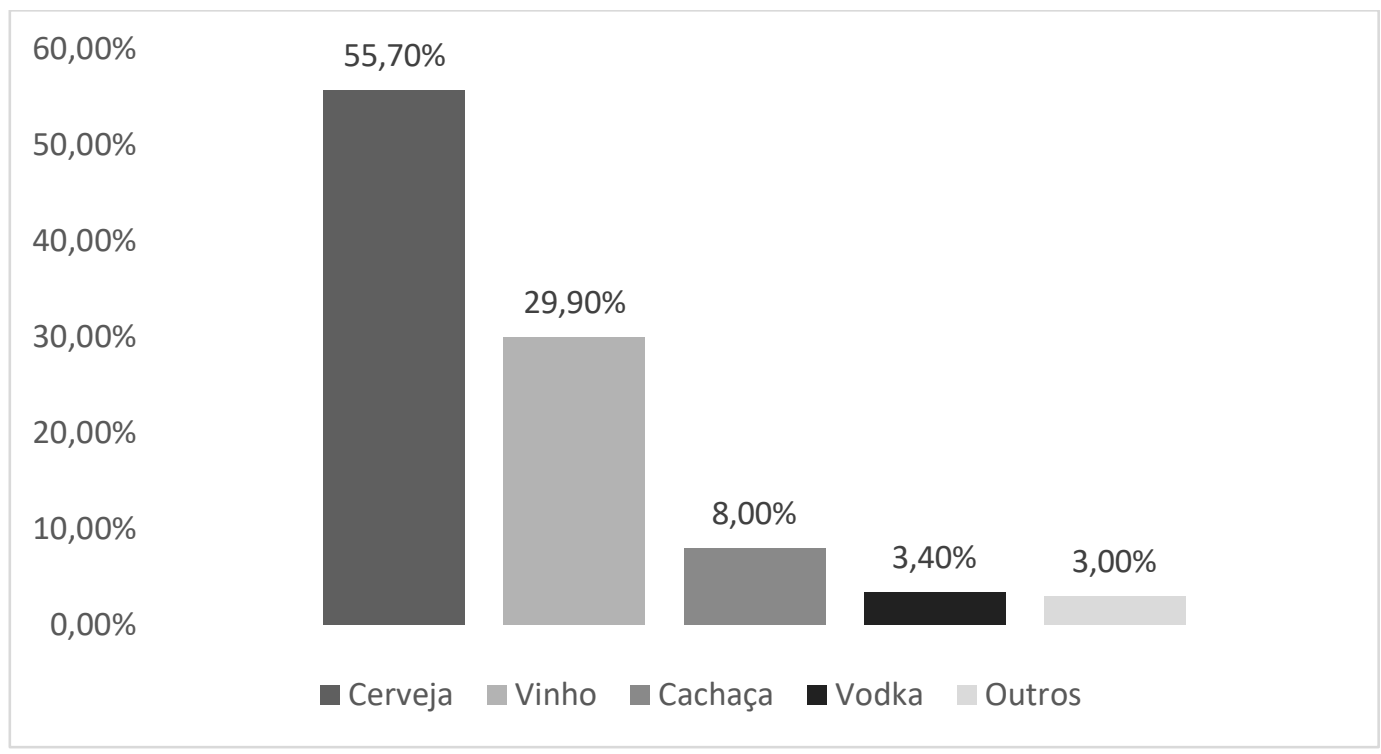

Fonte: Própria (2020)

Observa-se que, 36,2\% responderam que consomem bebida alcoólica uma vez por semana e que a cerveja é o tipo de bebida mais consumida (55,7\%), seguida pelo vinho (29,9\%). Pelicioli et al. (2017) ao avaliarem o perfil de 1.877 universitários brasileiros consumidores de álcool, verificaram que a frequência de consumo entre os respondentes variou de duas a quatro vezes ao mês (46,9\%) a mensal (40\%) e quanto ao tipo de bebida ingerida, houve predomínio de fermentados $(41,1 \%)$ em relação a destilados $(23,2 \%)$.

Na Figura 8 foi perguntado a todos os consumidores se eles sabem o que é uma bebida probiótica, 71,3\% responderam que sim. É possível notar uma crescente demanda por alimentos ditos como funcionais, ou seja, aqueles que segundo a ANVISA, quando consumidos como parte de uma dieta usual, produzem efeitos metabólicos e/ou fisiológicos benéficos à saúde, sendo considerados seguros para consumo sem supervisão médica (BRASIL, 1999).

Atualmente os alimentos funcionais são vistos como uma imagem positiva pelos consumidores, pelo fato de fornecerem benefícios à saúde sendo considerados como um novo caminho para uma alimentação saudável. Além disso, representam inovação comparada aos alimentos convencionais (COELHO, 2009).

Entre os vários micro-organismos considerados probióticos estão os gêneros Lactobacillus e Bifidobacterium pois são os gêneros que têm sido mais isolados da porção do trato gastrointestinal de um humano saudável (COELHO, 2009). Coppola e Turnes, (2004) comentam que também podemos considerar os gêneros Lactococcus, Enterococcus, Saccharomyces e Propionibacterium como espécies probióticas. Segundo Buriti e Saad, (2007) as espécies L. casei, L. Paracasei, L acidophilus e L. rhamnosus são de grande 
importância para a indústria de laticínios, devido ao seu emprego na produção de leites fermentados e na fabricação de queijos para a melhoria de qualidade do produto.

Figura 8. Conhecimento dos participantes sobre o que é uma bebida probiótica

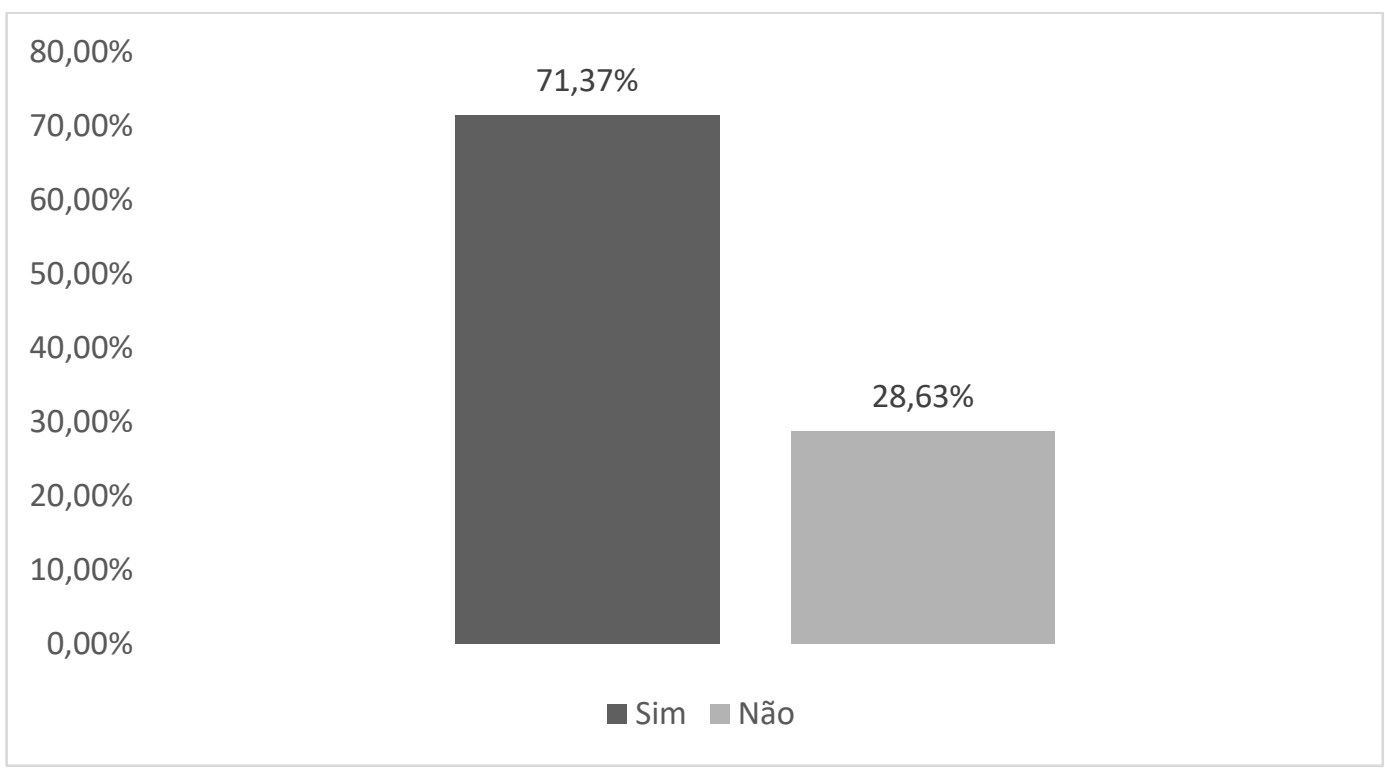

Fonte: Própria (2020)

Nas Figuras 9, 10 e 11 foram questionados se os participantes já ouviram falar da kombucha, se já a consumiram e se conhecem os benefícios associados à essa bebida, respectivamente. Em resposta, 62,59\% dos entrevistados já tinham ouvido falar da bebida, $44,27 \%$ afirmaram já ter consumido e 52,67\% disseram conhecer os benefícios kombucha.

Desde o seu descobrimento, existem relatos demonstrando que essa bebida pode auxiliar na redução do risco de doenças crônicas e também possui propriedades curativas, porém só na última década é que se observou uma explosão em sua popularidade principalmente em países como os Estados Unidos, que atualmente possui um mercado bem estabelecido para esse produto (SANTOS, 2016). Entretanto, ainda não existem comprovações científicas de seus reais benefícios, mas sabe-se que é uma bebida que apresenta grande potencial biotecnológico.

Um dos benefícios já estudados é a sua atividade antioxidante, relacionada com o aumento da imunidade, alívio de inflamações e artrites. Jayabalan et al. (2008) relataram a potencial habilidade de eliminação de radicais livres pelo chá de kombucha preparado a partir de chá verde e chá preto. Segundo os autores, os compostos fenólicos, a atividade de reação com radical DPPH e o radical superóxido foram aumentados ao longo do tempo de fermentação, enquanto o poder de redução, a capacidade de eliminação de radicais hidroxílicos e a capacidade de peroxidação antilipídica foram reduzidos. 
SOUSA, et al.

Figura 9. Participantes que já ouviram falar da kombucha.

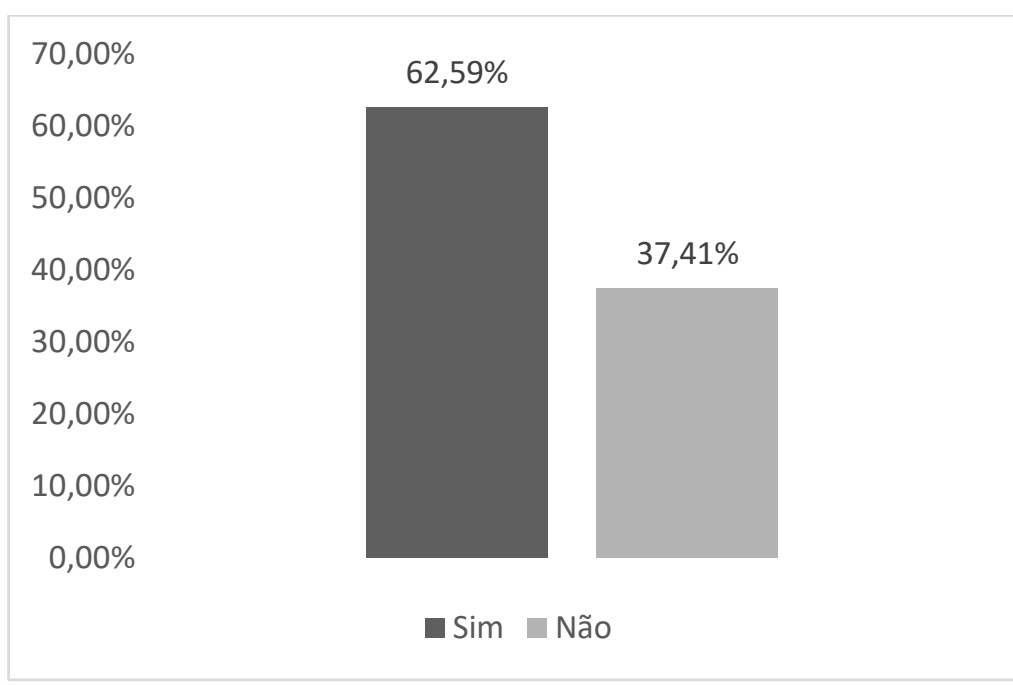

Fonte: Própria (2020)

Figura 10. Participantes que já consumiram a kombucha.

\begin{tabular}{|l|l|}
\hline $60,00 \%$ & $55,73 \%$ \\
$50,00 \%$ & $44,27 \%$ \\
$40,00 \%$ & \\
$30,00 \%$ & \\
$20,00 \%$ & \\
$10,00 \%$ & \\
$0,00 \%$ & - Nim Não \\
\hline
\end{tabular}

Fonte: Própria (2020)

Figura 11. Conhecimento dos benefícios associados à saúde pelo consumo de kombucha.

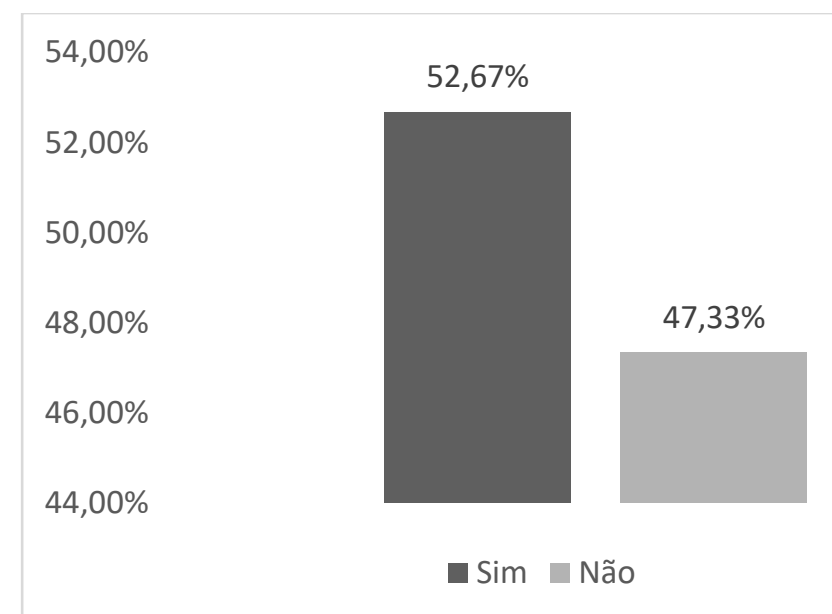

Fonte: Própria (2020) 
Na Figura 12 foi questionado se os participantes conheciam o processo de fabricação da kombucha. Observa-se que apenas 50,76\% disseram saber, enquanto 49,24\% responderam que não sabem como a bebida é produzida. A produção de kombucha ocorre a partir da infusão de chá verde ou preto adoçado com açúcar, que é substrato da reação de fermentação. As características sensoriais e a composição química da kombucha são dependentes de fatores como o tipo de chá e açúcar utilizado para o procedimento, dos microrganismos presentes no SCOBY (visto que a sua composição varia de produtor para produtor), além das condições de fermentação, como tempo e temperatura. O tempo de fermentação pode variar de 7 a 12 dias, considerando que quanto mais tempo o produto ficar fermentando mais ácido será o sabor, enquanto a temperatura pode variar de 22 a $30^{\circ} \mathrm{C}$ (DUFRESNE e FARNWORTH, 2000).

Estudos reportam que o espectro microbiano da bebida é dominado por bactérias ácidoacéticas e leveduras (CHAKRAVORTYA et al., 2016). Sabe-se que a composição microbiana da cultura do chá de kombucha varia de uma cultura para outra, dependendo de fatores como a localização geográfica, o clima, as espécies locais de bactérias e leveduras e a origem do inóculo (WATAWANA et al., 2015).

Figura 12. Conhecimento sobre o processo de fabricação da kombucha por parte dos participantes.

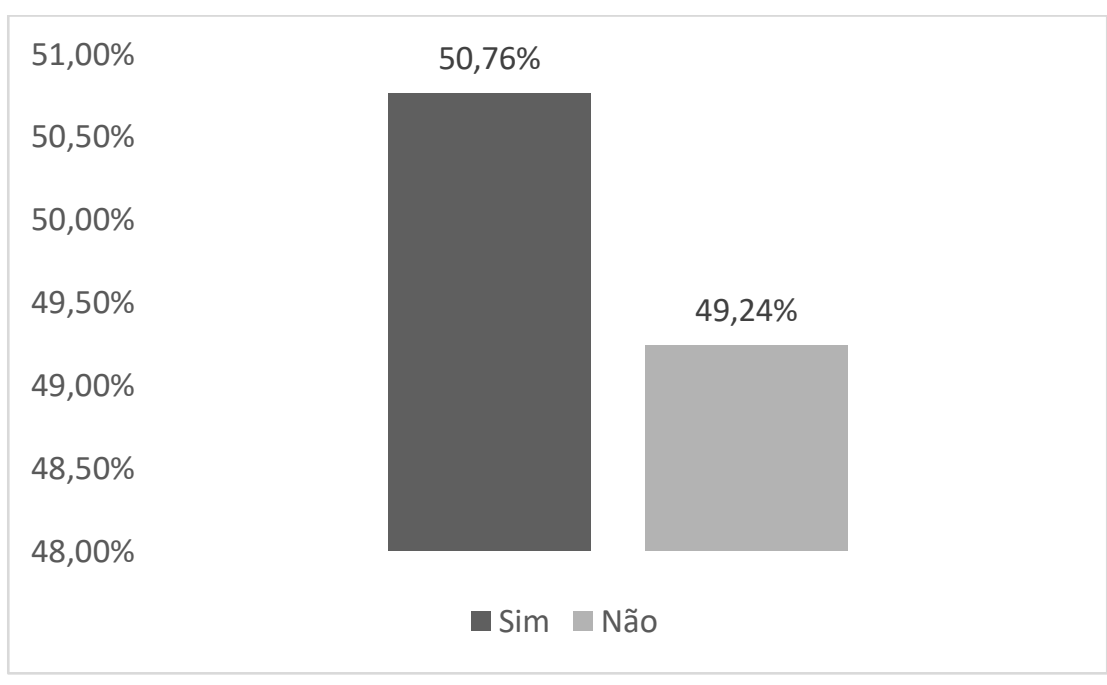

Fonte: Própria (2020)

A Figura 13 refere-se à disponibilidade da kombucha nos mercados consumidores. A maioria dos participantes $(79,77 \%)$ responderam que essa bebida não é facilmente encontrada. A kombucha ainda está em processo de popularização no Brasil e atende perfeitamente às atuais tendências do mercado.

Segundo a Brasil Food Trends (2020), a tendência denominada "saudabilidade e bemestar" vem desencadeando diversos segmentos de consumo, entre os quais é possível destacar 
a procura de alimentos funcionais, que têm como diferencial trazer benefícios ao desempenho físico e mental, saúde cardiovascular, saúde intestinal, entre outros.

Os probióticos ainda possuem sua aplicabilidade muito limitada à indústria de laticínios, sendo a retentora de grande parte do mercado de alimentos funcionais, e é de fundamental importância o desenvolvimento de novos produtos adicionados dessas culturas para a ampliação desse mercado e aceitação por novos consumidores, pois não são todos que podem ou apreciam produtos lácteos (COELHO, 2009).

Figura 13. Disponibilidade da kombucha nos mercados consumidores

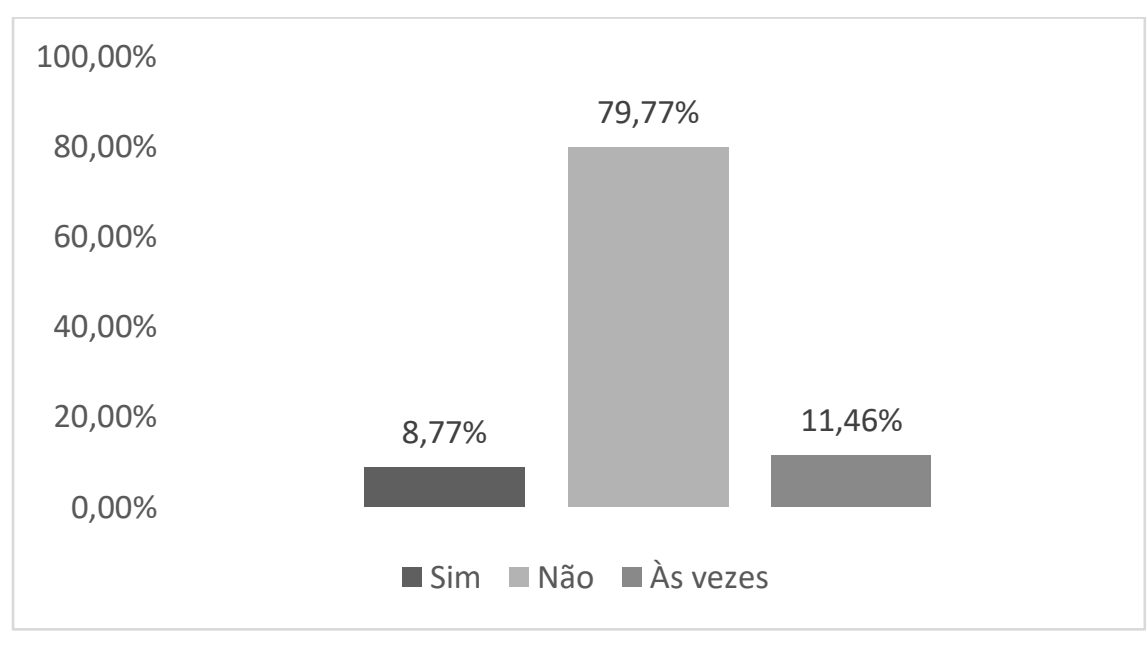

Fonte: Própria (2020)

\section{Conclusões}

Os respondentes são em sua maioria mulheres, entre 21 e 30 anos, apresentam pós-graduação e renda mensal entre 1 e 2 salários mínimos. Os participantes fazem o uso de álcool ao menos uma vez por semana, sendo a cerveja a bebida mais consumida.

A maioria dos respondentes afirmam saber o que é uma bebida probiótica e conhecem os benefícios associados à kombucha, no entanto, essa bebida não apresenta uma disponibilidade no mercado consumidor apesar de se mostrar bastante promissora no segmento de alimentos funcionais.

\section{Referências}

ASSOCIAÇÃO NACIONAL DOS EXPORTADORES DE SUCOS CÍTRICOS. Produção de Kombucha se multiplica no Brasil. São Paulo: CITRUS, 23 out. 2018. Disponível em: http://www.citrusbr.com/noticias/?id=312621. Acesso em 3 de julho de 2020. 
BRASIL. Portaria n ${ }^{\circ} 398$ de 30 de abril de 1999. Estabelece as diretrizes básicas para análise e comprovação de propriedades funcionais e ou de saúde alegadas em rotulagem de alimentos. Brasília: ANVISA, 1999.

BRASIL. Portaria $n^{\circ} 103$ de 20 de setembro de 2018. Instrução Normativa que visa estabelecer em todo território nacional o padrão de identidade e qualidade de kombucha. Diário Oficial da União, Brasília, n. 188, seção 1, p. 18, 28 set. 2018.

BRASIL Food Trends 2020. São Paulo: FIESP; ITAL, 2010. Disponível em: http://www.brasilfoodtrends.com.br/. Acesso em 04 de julho de 2020.

BRASIL. Instrução Normativa $n^{0} 41$ de 17 de setembro de 2019. Estabelecer o Padrão de Identidade e Qualidade da Kombucha. Disponível em: http://www.in.gov.br/en/web/dou//instrucao-normativa-n-41-de-17-de-setembro-de-2019-216803534. Acessado em 15 de julho de 2020.

BURITI; F. C. A; SAAD, S. M. I. Bactérias do grupo Lactobacillus casei: caracterização, viabilidade como probióticos em alimentos e sua importância para a saúde humana. Arquivos latino americanos de nutrição, v. 57, n. 4, p. 373 - 380. 2007.

CHAKRAVORTYA, S.; BHATTACHARYA, S.; CHATZINOTAS, A.; CHAKRABORTY, W.; BHATTACHARYA, D.; GACHHUI, R. Kombucha tea fermentation: Microbial and biochemical dynamics. International Journal of Food Microbiology, v. 220, 63-72, 2016.

CHEN, C.; LIU, B. Y. Changes in major components of tea fungus metabolites during prolonged fermentation. Journal of Applied Microbiology, v. 89, n. 5, p. 834-839, 2000.

COELHO, J. C. Elaboração de bebida probiótica a partir do suco de laranja fermentado com Lactobacillus casei. Orientadora: Sueli Rodrigues. 2009. 95 f. Dissertação de Mestrado (Ciências e tecnologia de alimentos) - Universidade Federal do Ceará, Fortaleza, 2009.

COPPOLA, M. M.; TURNES, C.G. Probióticos e resposta imune. Ciência rural, v. 34, n. 4, p. $1297-1303.2004$. 
DUFRESNE, C.; FARNWORTH, E. Tea, Kombucha, and health: a review. Food Research International, v. 33, n. 6, p. 409-421, 2000.

JAYABALAN R. et al. Changes in free radical scavenging ability of kombucha tea during fermentation. Food Chemistry, v. 109, n. 1, p. 227-234, 2008.

MARTINS, D. Comportamento do consumidor de cervejas artesanais no município de Santana do Livramento. Orientador: João Garibaldi Almeida Viana. 2019. 25 f. Trabalho de conclusão de curso (Bacharelado em Administração) - Universidade Federal do Pampa, Campus Santana do Livramento, Santana do Livramento, 2019

PELICIOLI, M et al.. Perfil do consumo de álcool e prática do beber pesado episódico entre universitários brasileiros da área da saúde. J Bras Psiquiatr., v.66, n.3, p.150-156, 2017.

RIBEIRO, E. S. S. Elaboração e caracterização de bebida probiótica a partir de suco de cajá fermentado Lactobacillus acidophilus NRRL B-4495. Orientado: Francisco Canindé Sousa Junior. 2020. 86 f. Dissertação de Mestrado ( Nutrição) - Universidade Federal do Rio Grande do Norte, Natal, 2020.

SANTOS, M. J. Kombucha: caracterização da microbiota e desenvolvimento de novos produtos alimentares para uso em restauração. Orientadora: Catarina Prista. 2016. $119 \mathrm{f}$. Dissertação de Mestrado (Ciências Gastronômicas) - Universidade Nova de Lisboa, Lisboa, 2016.

WATAWANA, M. I.; JAYAWARDENA, N.; GUNAWARDHANA, C. B.; WAISUNDARA, V. Y. Health, Wellness, and Safety Aspects of the Consumption of Kombucha. Journal of Chemistry, article ID 591869, p. 1-11, 2015.

YASSIN, L. S. Desenvolvimento de Smoothie fermentado por microorganismos da Komucha com base no perfil de consumo, composição química, compostos bioativos e descrição sensorial. Orientador: Alessandro Nogueira. 2019. 112 f. Tese de Doutorado ( Ciência e Tecnologia de Alimentos)- Universidade Estadual de Ponta Grossa, Ponta Grossa, 2019. 